\title{
Assess the performance of the diagnosis ways of diabetic retinopathy
}

\author{
Mohammad Q. Jawad ${ }^{1}$, Maryam A. yousif ${ }^{2}$, Dina H. Abbas ${ }^{3}$ \\ ${ }^{1}$ University of Information Technology and Communications \\ ${ }^{2,3}$ University of Baghdad, College of Science for women, Computer Science Department
}

\section{Article Info}

Received January $18^{\text {th }}, 2019$

\section{Keyword:}

Exudates,

Fcm clustering,

Image processing,

morphological operations,

optic disk detection,

Fundus image,

Diabetic retinopathy (dr),

Macula

\begin{abstract}
Considered the diagnosis of diseases using image processing is one of the most important areas of image processing techniques used in the medical field, where is the digital data in the field of ophthalmology focus of researchers for automatic detection of some important diseases such as diabetic retinopathy (DR).

And is defined as damage to the retina of the eye comes as serious complications and on the human body complications resulting from diabetes in the long term and is considered one of the most important causes of blindness in the world and cause serious damage to the retina.

The research aims to Assess the performance of some of the methods used in the diagnosis of diabetic retinopathy by revealing one of the most important accompanying pests him in the retina of the eye and is the exudates and through diagnosed in images digital fundus through image processing techniques where this detection process contributes in helping to early detection.
\end{abstract}

\section{Corresponding Author:}

Mohammad Q. Jawad

University of Information Technology and Communications

Email: mohammad.qassim2002@uoitc.edu.iq

\section{Introduction:}

Diabetes has become a global epidemic, with the number of people infected with PU rising from 108 million in 1980 to 422 million in 2014, equivalent to $8.5 \%$ of the adult population over the age of 18 in the world. It will be one of the seven leading causes of death in 2030. One of the most important features of diabetes is a complication of the retinal microvasculature and approximately $2.6 \%$ of the causes of blindness in the world are due to diabetic retinopathy [01].

Figure (1a) and (1b) show a toxic retina that includes identification of important parts of the retina such as the optic disc, macula and fovea, and others with some form of diabetic retinopathy that manifested secretion and hemorrhage respectively. 


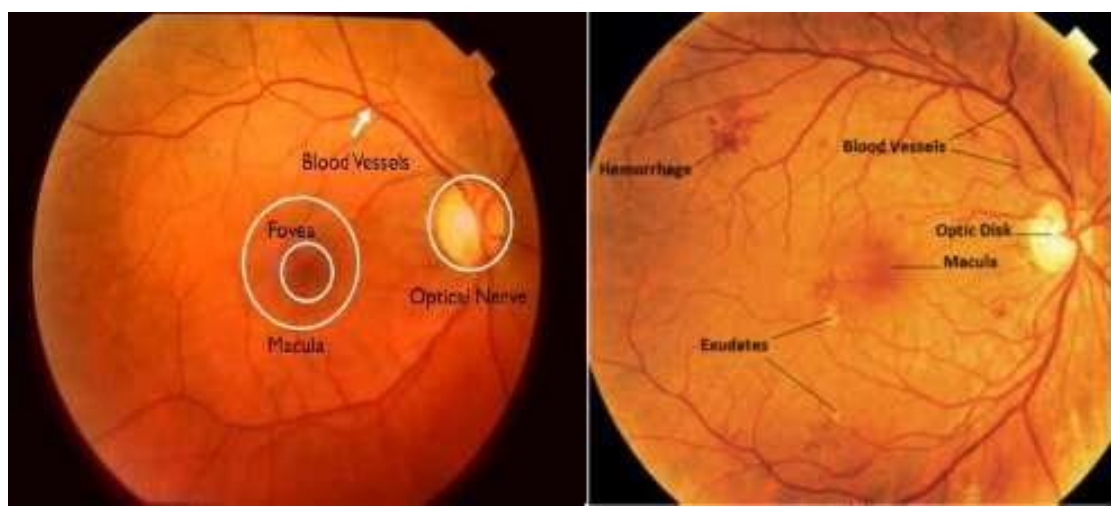

(a)

(b)

Figure 1. - (a) uninfected toxic eye - (b) eye with diabetic retinopathy

Diabetic retinopathy is detected by processing fundus images using various techniques

Techniques in the field of vasculature of the fundus and fragmentation of the framework Fraz, which classified algorithms for extraction of blood vessels [21,1].

Optical disk removal techniques:

Approach the optical disk by identifying the largest protected contrast of a window size of $80 * 80$ through the Sinthanayothin hypothesis, which is based on the fact that those with retinal lesions will experience less variation in the area of the optical disk [2].

1- Detection of the optical disk through the entropy filtering through the suggestion [3] Sopharak

- Niemeijer detects the optical disk by adopting a set of characteristics of the depth of the eye such as the map of blood vessels in the eye and image density such as the number of blood vessels and the average width lim and standard deviation and direction and others [4].

The mechanism of proactive detection of secretion for the diagnosis of diabetic retinopathy is carried out through various methods:

2- Sopharak method based on the use of a set of morphological and infectious generalizations [21,5].

[6] Fuzzy C-Means clustering based on Sopharak technique.

2 - Adoption of Ramasway Comparing the different techniques of the detector for the release of morphological approach, which is a set of nonlinear generalizations associated with the element or the characteristics of the photographer and processes the morphology of the image through a small form called a picture element or element Yecm The region growing approach is a simple technique that depends on the selection of pixels from the image or a group of pixels and considering the area and then study the bicycles adjacent to the region, if it was similar to the region was added and thus the region grows, if not similar to the characteristics of the region. It is excluded and s For example, the properties adopted are fuzzy C-mean Clustering, which studies each part of the data, and the possibility of belonging to two or more specific groups of the comparison process, called clusters, and determines that part of the Data for a cluster based on the distance between the center of the cluster and the data point, and the shorter the distance, the more the part belongs to that cluster, and k-means clustering, a technique that relies on grouping data into groups (clusters) with the same fuzzy C-mean clustering technique. The only difference is that the point studied in k-means clustering either belongs to a cluster or not a tin [7] While the ANU is characterized by fuzzy clustering, the studied point can belong to more than one cluster with varying affiliation ratios [7]. 


\section{The Importance and Objectives}

\section{of the Research The importance of the research lies in:}

Provide a practical technique that detects the early detection of diabetic retinopathy and diagnosis in the first Marhmu because the development of wider and close to the macula in the eye can lead to loss of vision and blindness

To provide a comparison between some of the methods used in the field of image processing for the detection of diabetic retinopathy

Research Methods and Materials

The proposed method for the detection of a fundus-secretion detector for the diagnosis of diabetic retinopathy relies on performing a set of steps shown in Figure 2 and performing preliminary treatment on the fundus image.

The optical disc is detected and then removed. In the end, the secretion is detected using the FCM algorithm, and images from the online Diaretdb0 and Diaretdb1 databases have been used [11].

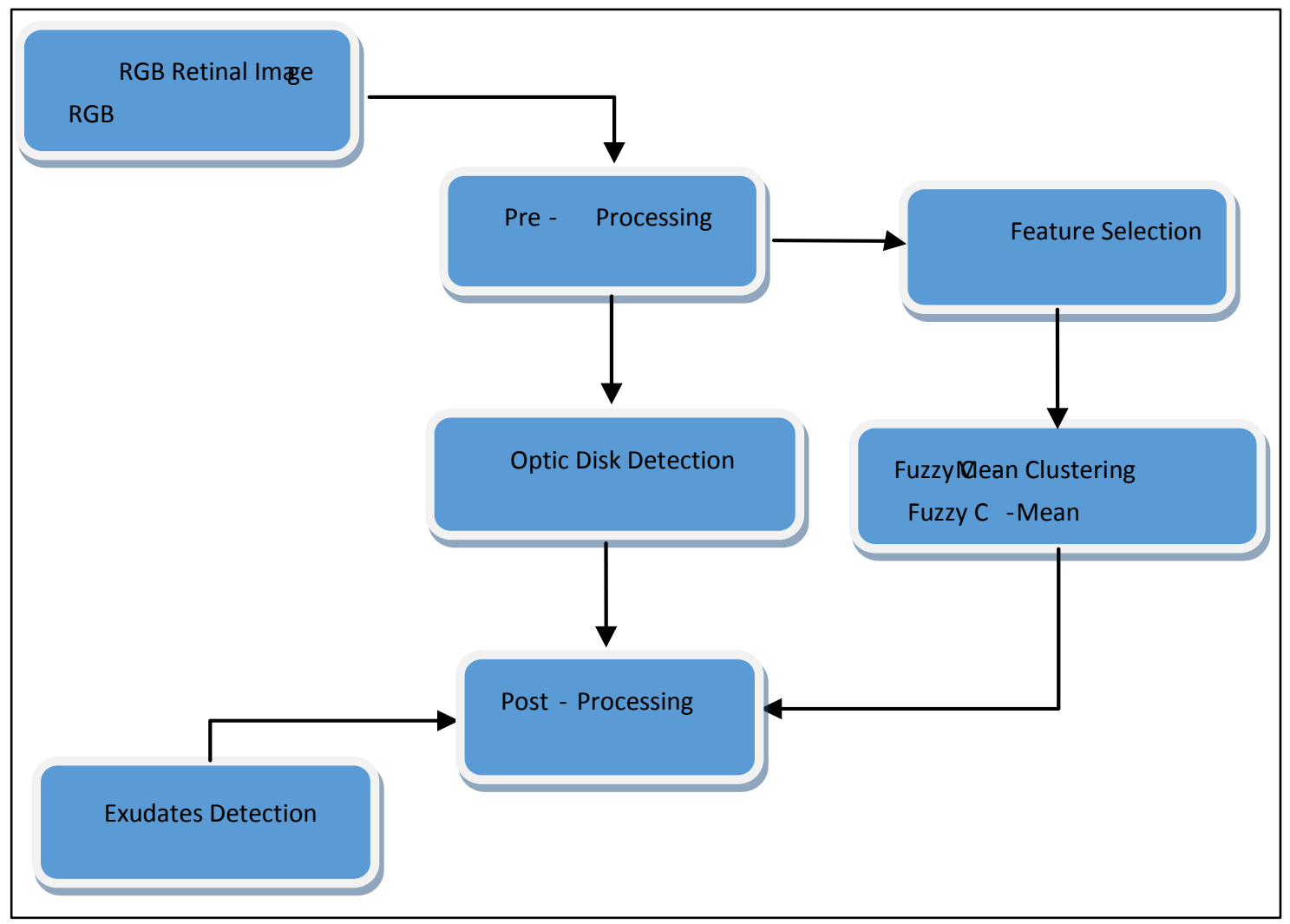

Figure 2. Sequence of the proposed steps for the detection of secretions in the fundus images and the following is a land enabling steps in detail.

Initial blindness of eye fundus

These initial operations are the first step in the prognostic diagnosis of retinal motherland and is a sensitive stage in the preparation of fundus images including the following steps:

1 - Resize the fundus images to 400 pixels $* 450$.

2 - Extract the green channel from the RGB image because it provides maximum protected contrast over the entire pixel of the image. 


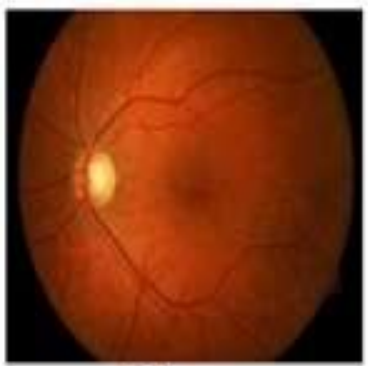

(a)

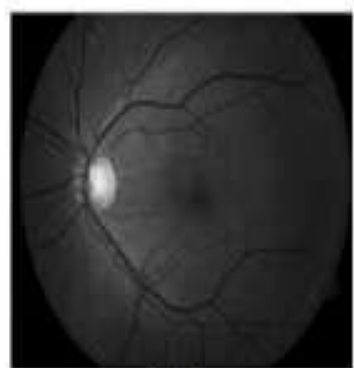

(b)

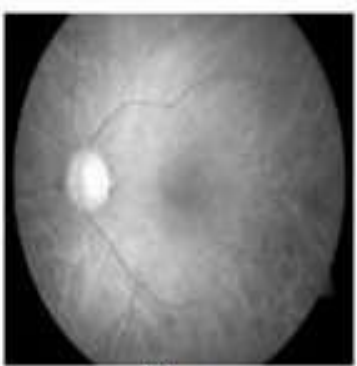

(c)

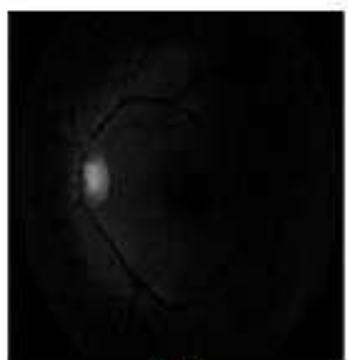

(d)

Figure 3. Shows the picture of the fundus and the three mononal channels

3 - Apply a median filter size of $3 * 3$ pixels in order to return the median values of elements over the entire dimensions of the different matrix and does not work on blurring the boundaries of the image and Figure (4) shows the result of the application of this period

4 - Apply an image contrast equalizer using a contrast-limited adaptive histogram equalization (CLAHE) to enhance image contrast where CLAHE operates on small areas of the image called patches instead of the whole image. The bilinear interpolation of industrial boundaries can vary, especially in homogeneous areas, to avoid any noise that may be present in the image, and the retinal image resulting from the previous processes is shown in Figure 4.

2 - optical disk detection

The optical disc possesses a circular shape and is characterized by an area of high density that looks bright and has a yellow color. It may be diagnosed as part of the FRZ region due to the mono-chapo with the FRZ so it must be detected and isolated so that we can determine the presence of FRZ The optical disk OD was detected using Saleh's idea by increasing the steps and resizing the element of the yumma [8].

In this step we adopted the following steps:

1 - The application of Top-Hat morphological coefficient on the image based on the SE element and a ball element with a 48-pixel radius and a height of 55 pixels will be used. Figure 4 shows the image after the Top-Hat operation.

2 - Apply the contrast spread to the image. This function extends the range of density values of the image and apply the contrast spread by specifying the minimum and maximum possible blindness of the image. By default, the minimum and maximum values are set at $1 \%$ down and $\% 2.1$ Blinds all pixel values and shows the resulting image as shown in Figure 4. (f)

3 - Convert the resulting image to a binary image using a threshold value or level of 0.9 and threshold values in the field 1 [, 0], which is relative to the possible signal levels of the image class and therefore a value level of 0.5 is median between black and white regardless of Layer, where the resulting output image replaces all the pixels in the input image with illumination values greater than the level by 1 (white) and the rest of the pixels by 0 (black), and the image is shown after the operations as shown in Fig. 4 (g).

4 - The use of morphological parameters Opening and Closing, where the Opening coefficient is used to spend on small elements and the closing coefficient is applied using the disk structure element with a diagonal circle 10, and the resulting image after the application of these two processes in Figure (h) 4, and after the application of all The previous phases will remain on the optical disk and after detection, the retina image will be deleted 


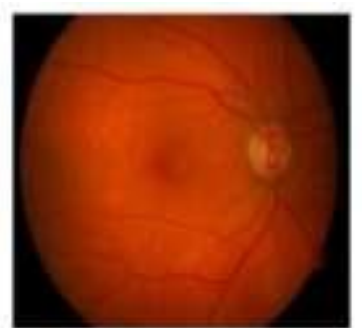

(a)

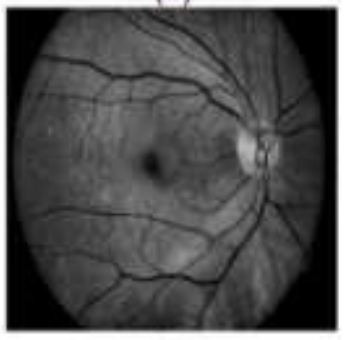

(d)

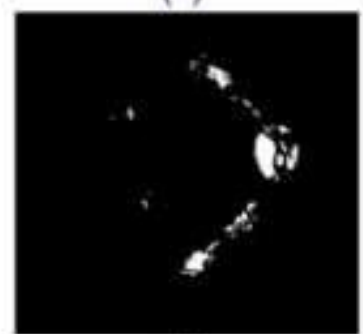

(g)

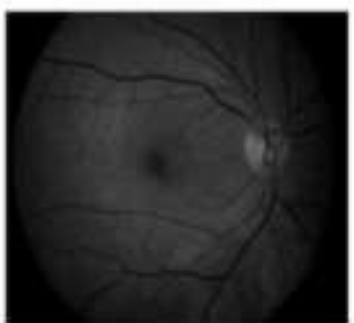

(b)

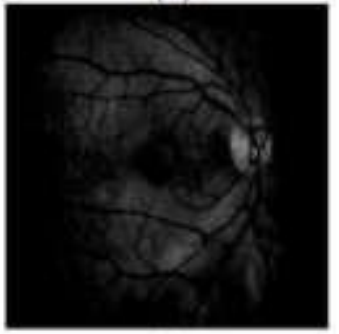

(e)

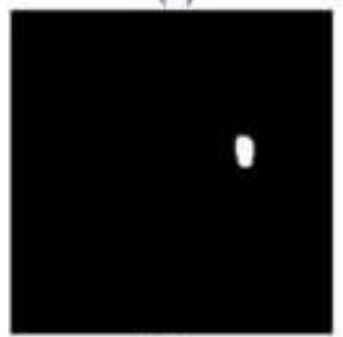

(h)

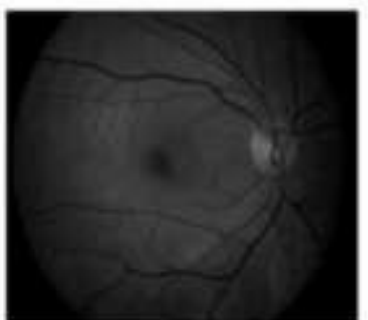

(c)

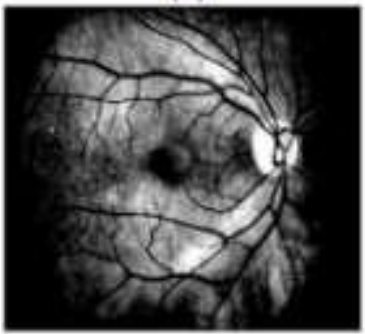

(f)

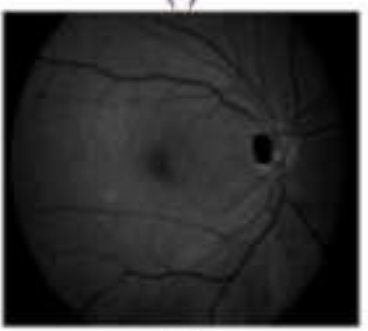

(i)

Figure 4. Optical Disc Detection (a) Main Image (b) Vegetable Channel (c) Filter (d) median Contrast Equation (e) Coefficient (f) Top-Hat Contrast Expansion (g) Binary Image (h) binary (I) Opening and Closing The optical disk is deleted

Figure 5 shows the element of the proposed optical disk heap with a diameter of 3 pixels.।

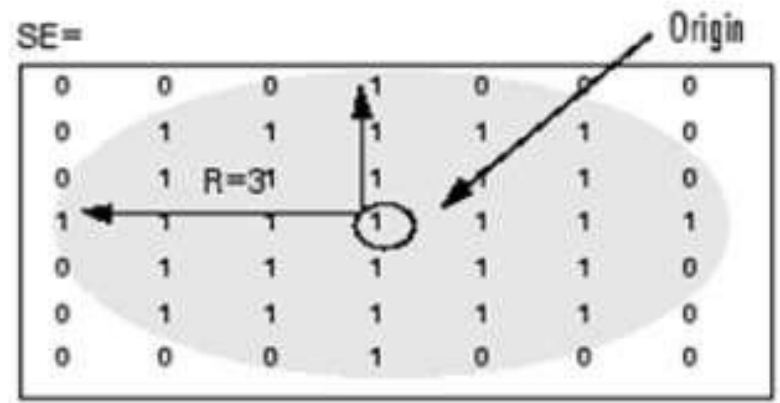

Figure 5. The disk heap component

\section{The phase-out phase}

Objects were loaded in front of the cryptocurrency more liquid if the cryptochemistry was removed and the resulting image after cryptocytosis was removed in a later step as another feature in the secretion detection process. Hat according to the following steps:

1 Calculation of Top-Hat operation blindness of the image and the resulting image is shown in Figure (b) 6 
2 Bottom-Hat is a combination of Closing and minus, where Bottom-Hat calculates the morphological Closing coefficient on the image and then subtracts the original image based on the element of the image resulting from the Closing process. A disc with a diameter of 5pixels, and Figure (c) 6 shows the resulting image after the second step in the phase-out process and the BottomHat coefficient.

Subtract the image resulting from the Bottom-Hat process from the image resulting from the process of collecting the green channel of the main photographer and the resulting image after the Top-Hat operation and the result is shown in Figure (6)

subtract the resulting image from the median image filter size $25 * 25$ pixels where the resulting image appears after Apply a median filter in Fig. 6 and a subtraction image is shown in Fig. 6.

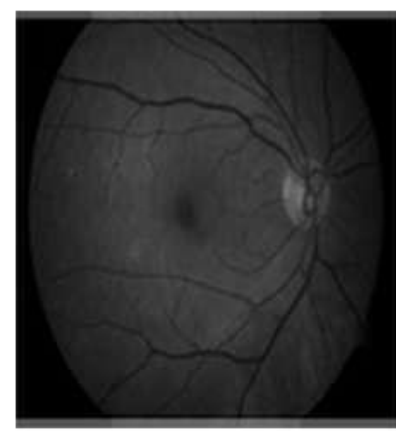

(a)

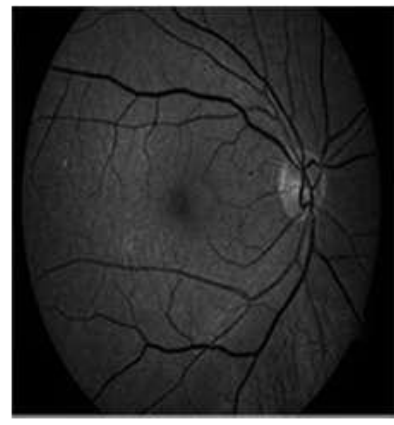

(d)

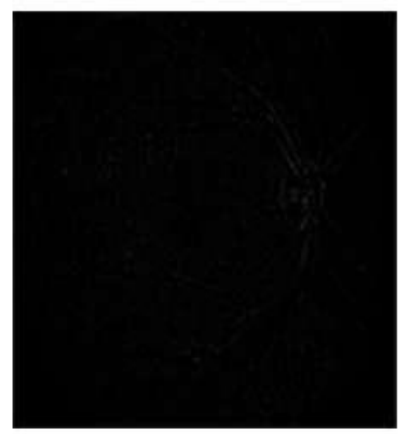

(b)

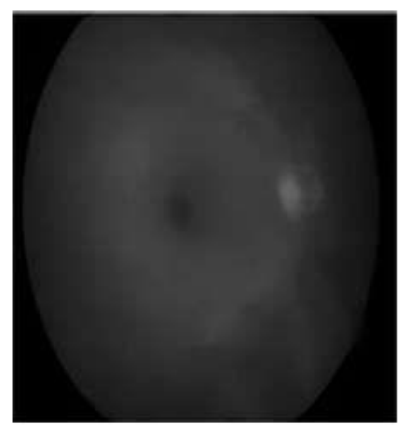

(f)

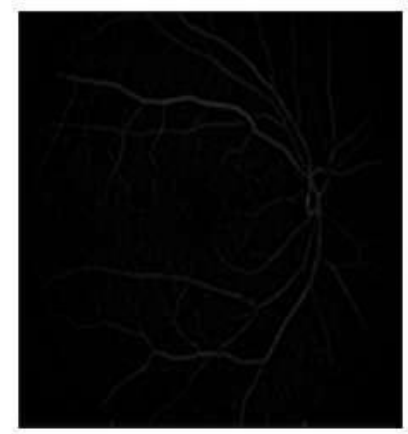

(c)

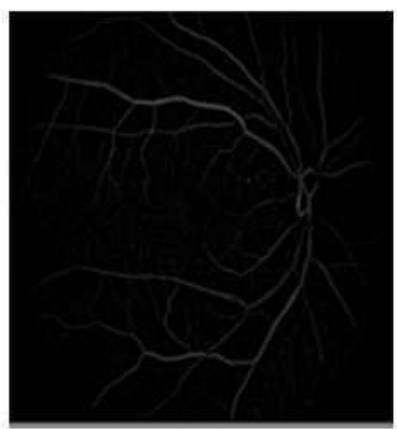

(g)

Figure 6. (a) the green channel of the main picture (b) coefficient (c) Top-Hat coefficient (e) $[(a+b)-(c)](d)$ Bottom-Hat filter (f) Median $25 * 25$ image delete hidden

\section{Detection of secretions}

The FCM algorithm is used to diagnose the secretion of retinal images. The FCM algorithm adopts the Fuzzy C-means clustering method based on a specific data set [9].

1. (FCM clustering) FCM Assembling .2.4

A bloated aggregation algorithm that employs a fuzzy division where a given data point can belong to two or more clusters with specific affiliations with a membership rank between 0 and 1 .

The input media for the FCM function are the data set and the number of clusters, while the output is the list of cluster positions and $n$ degrees of membership for each pixel where $n$ is the number of clusters required. 5 .

Extract the mezzanine

Four advantages were selected for use as input for the FCM algorithm

- Remove the hidden image.

- HV channel of retinal image HSV format.

- V-channel of HSV format for retinal image. 
- Standard deviation of the green channel from the retinal image.

The monochrome RGB space of the dummy image was initially converted to Hue (HSV, Saturation, and Value) of the image where Hue and Value were extracted from the HSV format of the retina image because the secretion possesses zero yellow colors and Figure 7 (a) extracts the channel out. Hue of the retina image and Figure (b) 7 shows the Value channel of the retina image.

The four feature is the standard deviation of the green channel of the retina image window size $3 * 3$ pixels and the result is shown in Figure (c) 7 and the image of deleting the hidden in Fig.

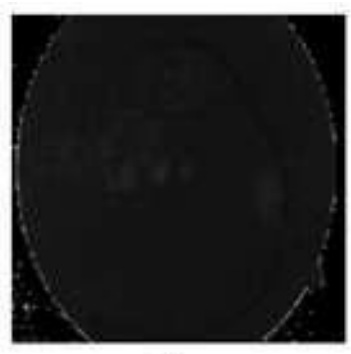

(a)

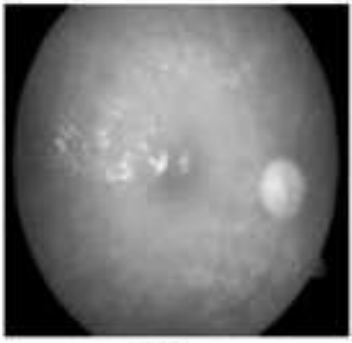

(b)

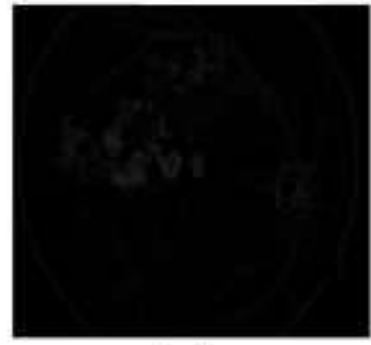

(c)

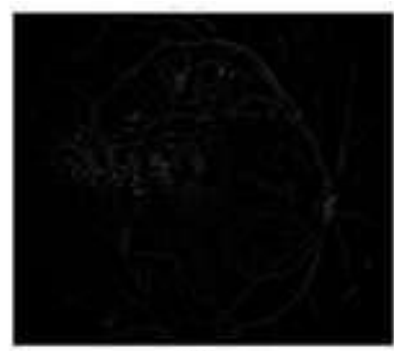

(d)

Figure 7. (a) Channel Extraction (b) Hue Channel Extraction Value

\section{(c) the standard deviation of the vegetable channel.}

After extraction of the mezzet, the next step was used as an input to the FCM algorithm of a classifier. The desired cluster was selected from 5 output clusters and the output clusters of the FCM algorithm were shown in Fig. 8.

In this step, the appropriate cluster is selected and after enhancing the contrast and deleting the optical disk, it is shown as a final output and the secretion area is detected in the retinal images.

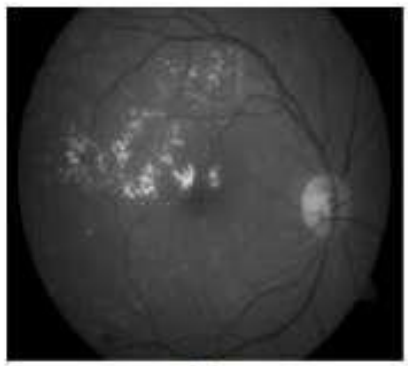

(a)

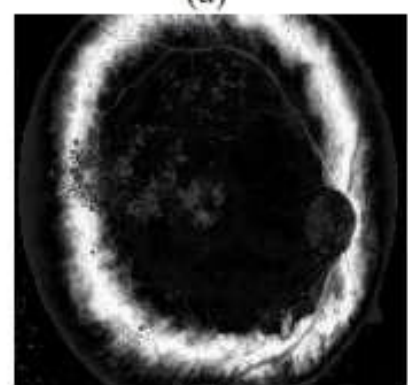

(d)

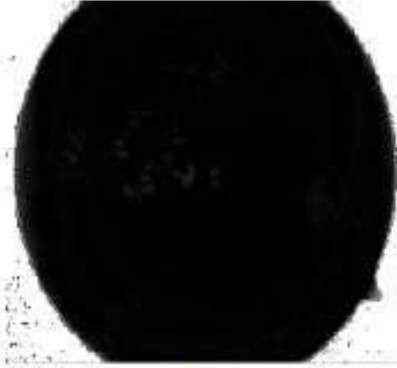

(b)

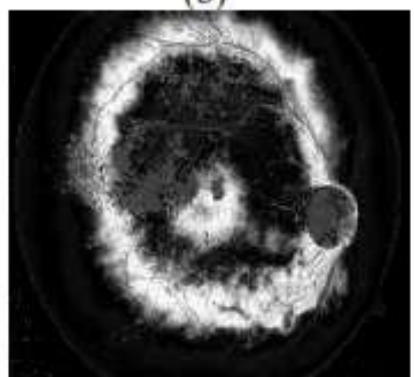

(e)

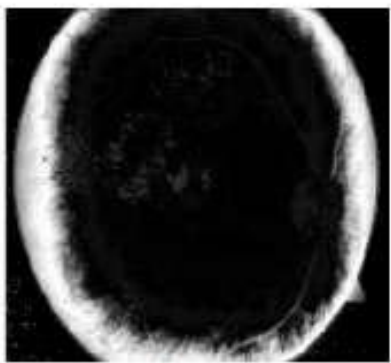

(c)

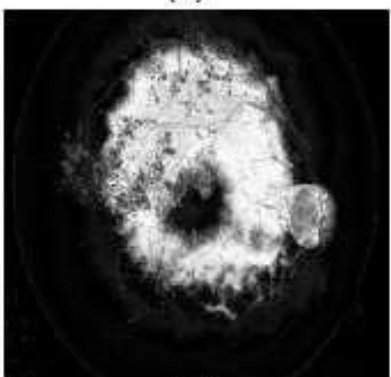

(f)

Figure 8. Cluster 5 (f) cluster4 (e) cluster3 (d) cluster2 (c) cluster1 (b) Main image (a)

M performance neck 
In this part of the research, performance was evaluated following the implementation of the proposed steps based on formulas for measuring performance:

positive predictive value: PPV and given by:

$$
P P V=\frac{T P}{T P+F P}
$$

TP: The number of correct pixel-secreting values detected.

FP: The number of pixel values that do not include Frazet and are incorrectly specified as Frazat places. Sensitivity is given by:

$$
\text { Sensitivity }=\frac{T P}{T N+F P}
$$

TN: The number of pixel values that do not include a fractal and are correctly detected as pixel values that do not contain a fractal.

Specificity and given in relation:

$$
\text { Specificity }=\frac{T N}{T N+F P}
$$

Accuracy is given by :

$$
\text { Accuracy }=\frac{T P+T N}{T P+F P+F N+T N}
$$

FN: The number of pixel excreted values not detected (specifically).

\section{Results and discussion}

The steps of the proposed method for the detection of secretion in the fundus images for the diagnosis of diabetic retinopathy were programmed using the MATLAB 2016a working environment and retinal images from the Diaretdb0 and Diaretdb1 databases. With true or false detection cases or without the discovery of a photographer from the Diaretdb0 databases

.Diaretdb1

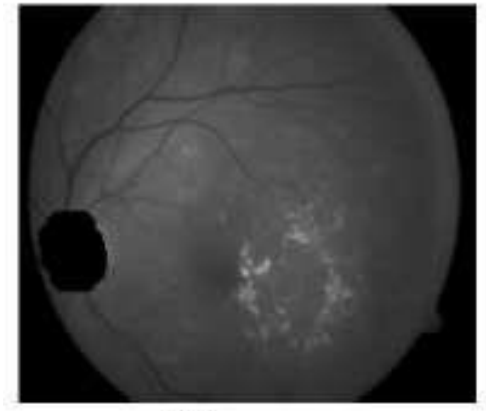

(a)

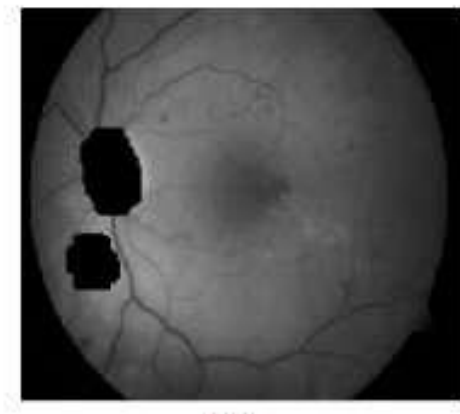

(b)

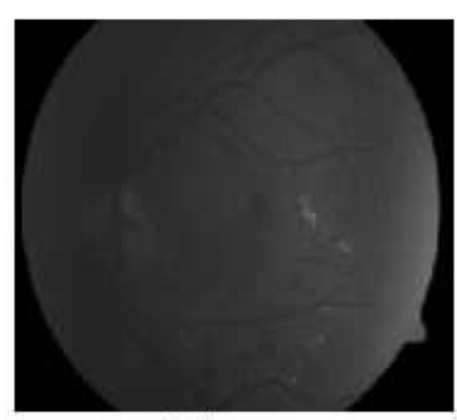

(c)

Figure 9. Optical disc detection (a) True detection (b) False identification (c) Non-detection

In some cases, the proposed method was unable to detect the optical disk due to the low contrast of retinal images, and in some cases the optical disk was incorrectly identified. 
The number of correct, false or unspecified instances of the optical disk was determined on the Diaretdb0 and Diaretdb1 databases.

Table 1. Optical disc detection performance

\begin{tabular}{|l|l|l|l|l|l|}
\hline $\begin{array}{l}\text { Retinal Images } \\
\text { Databases }\end{array}$ & $\begin{array}{l}\text { Number of } \\
\text { Images }\end{array}$ & $\begin{array}{l}\text { True } \\
\text { detection }\end{array}$ & $\begin{array}{l}\text { False } \\
\text { detection }\end{array}$ & $\begin{array}{l}\text { No } \\
\text { detection }\end{array}$ & $\begin{array}{l}\text { Average of true } \\
\text { detection of OD (\%) }\end{array}$ \\
\hline Diaretdb0 & 129 & 113 & 6 & 10 & 87.59 \\
\hline Diaretdb1 & 89 & 77 & 3 & 9 & 86.51 \\
\hline
\end{tabular}

Table (2) shows the comparison of the proposed working steps of the optical disk detector with other methods. It is important to note that the optical disc detection phase is only one part of the proposed steps. The features of the optical disk such as the circular and the starting point of the retinal blood vessels will improve the quantitative performance.

Table 2. Comparing the percentage of optical disk detection success in several ways

\begin{tabular}{|l|l|l|}
\hline Optic disc detection methods & Diaretdb0 & Diaretdb1 \\
\hline Sinthanayothin et al.(199)9 & - & 84 \\
\hline Lalond et al.(2001) & 77.56 & 75.46 \\
\hline Sopharak et atl.(2008) & 95.29 & 93.70 \\
\hline Ravishankar et al.(2009) & 80.12 & 76.41 \\
\hline Niemeijer et al.(2009) & 78.20 & 77.04 \\
\hline Proposed method & 87.59 & 86.51 \\
\hline
\end{tabular}

The proposed method of testing for the release of ten retinal images was also tested with the release of Diaretdb0 and Diaretdb1 databases. Data search.

Table 3. Average Proposed Detection Algorithm

\begin{tabular}{|l|l|l|l|l|}
\hline Method & Sensitivity (\%) & Specificity (\%) & PPV (\%) & Accuracy (\%) \\
\hline FCM & 88.66 & 99.98 & 95.66 & 99.90 \\
\hline
\end{tabular}

As illustrated at the beginning of the research, many methods using morphological and growth zone treatments and other methods are used to diagnose secretion of retinal images.

Sopharak's deliberate method of FCM showed a higher degree of sensitivity while the lowest was in positive conjecture value, while the Ramaswamy method based on the same FCM technique had results close to the proposed work steps in privacy and accuracy and outperformed sensitivity while weaker in positive conjecture value.

Table 4. Comparison of Effluent Detection Methods

\begin{tabular}{|l|l|l|l|l|l|}
\hline No. & Method & $\begin{array}{l}\text { Sensitivity } \\
(\%)\end{array}$ & $\begin{array}{l}\text { Specificity } \\
(\%)\end{array}$ & $\begin{array}{l}\text { IPPV } \\
(\%)\end{array}$ & $\begin{array}{l}\text { Accuracy } \\
\%)\end{array}$ \\
\hline 1 & Sopharak et al.(2)800 Morphology & 80.00 & 99.46 & - & - \\
\hline 2 & Sopharak et al.(2009) FCM & 97.2 & 85.4 & 5.9 & 85.6 \\
\hline 3 & Sopharak et al.(2008) FCM \& Morphology & 87.28 & 99.24 & 42.77 & 99.11 \\
\hline 4 & Ramaswamy et al.(2011), morphology & 82.23 & 99.95 & 40.93 & 99.18 \\
\hline 5 & Ramaswamy et al. (2011), FCM & 92.08 & 99.95 & 86.96 & 99.79 \\
\hline 6 & Ramaswamy et al. (2011), Region Growing & 82.23 & 99.93 & 42.70 & 99.31 \\
\hline 7 & Proposed Method,10 Images & 88.86 & 99.98 & 95.66 & 99.90 \\
\hline
\end{tabular}

The output images after the implementation of the proposed work steps are shown in Figure 9 and the output images after applying the optical disc detection algorithm as shown in Figure 10. 

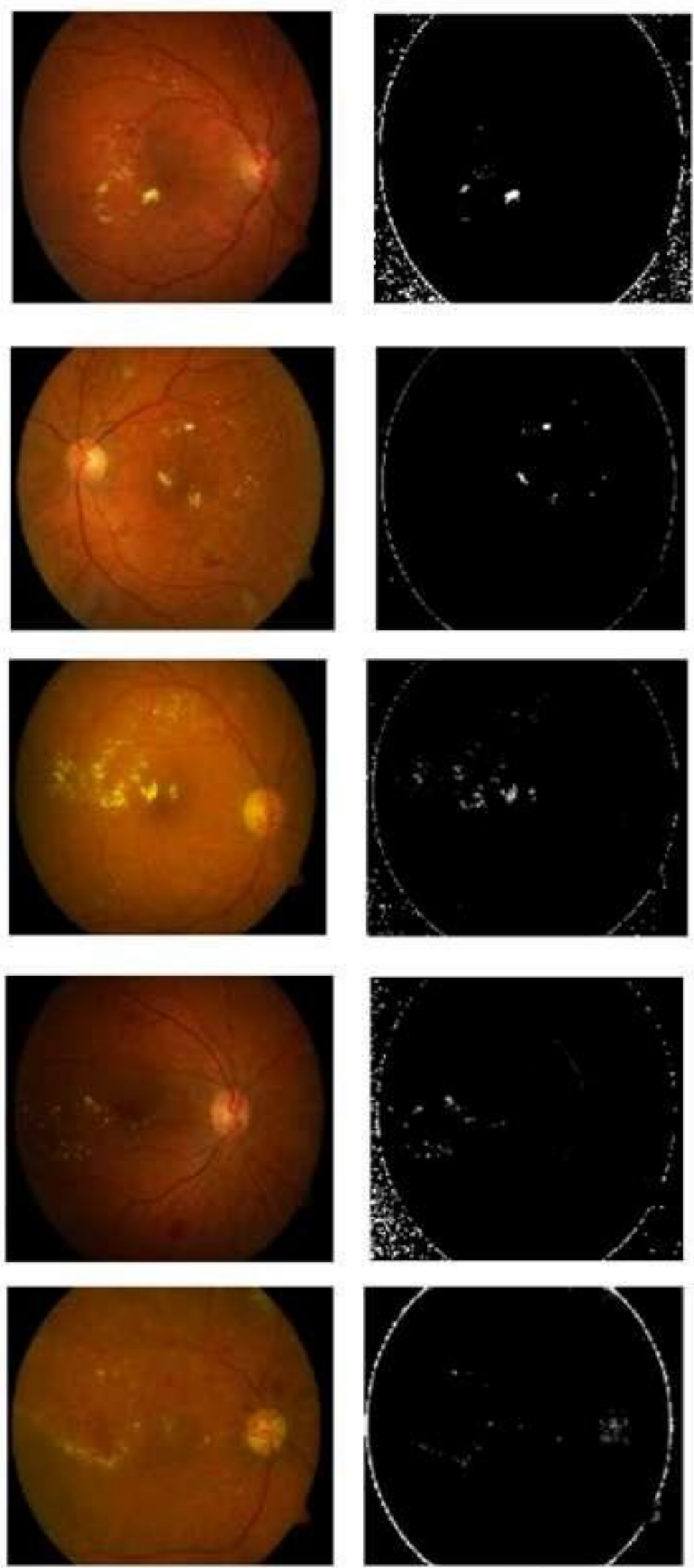

(a)

(b)

Figure 9. Show the Origin, Secretions, 

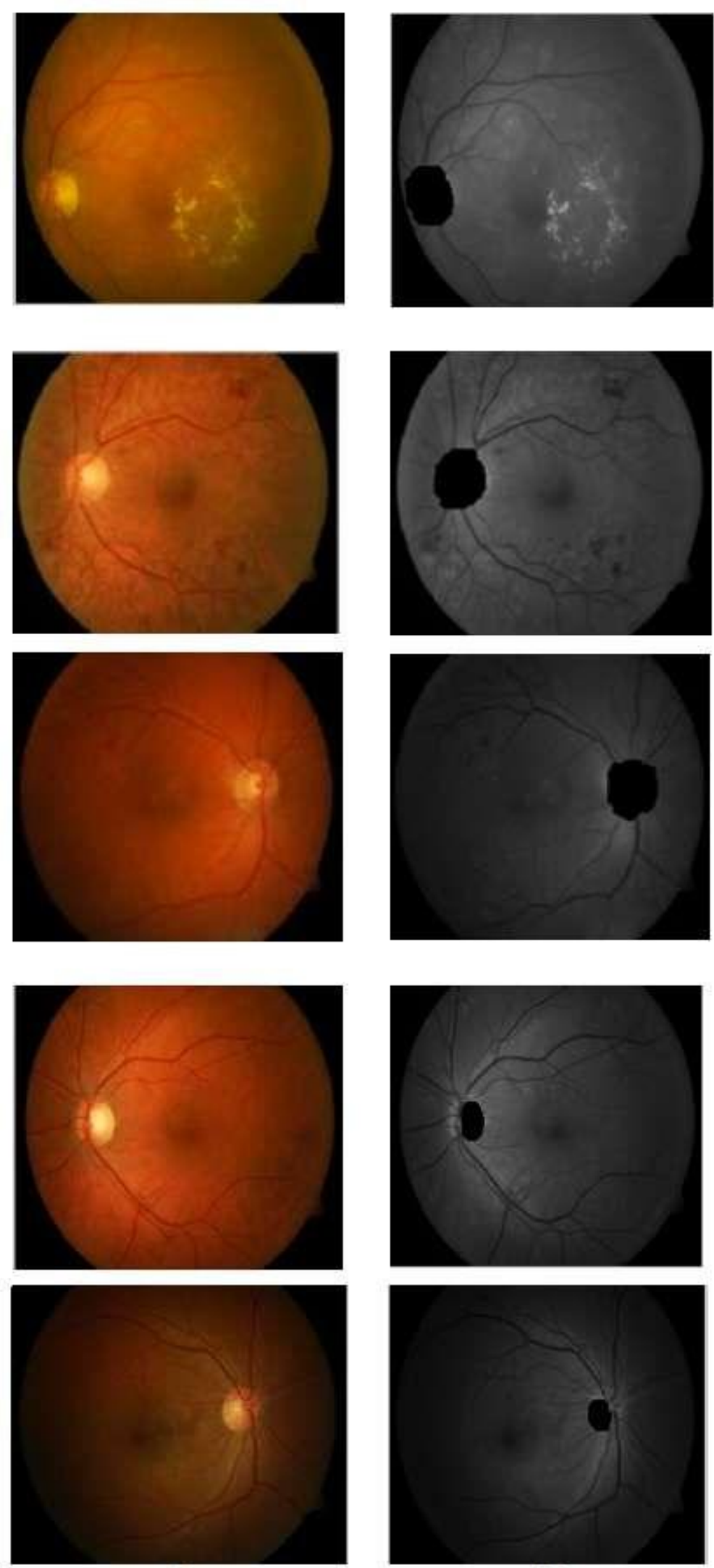

(a)

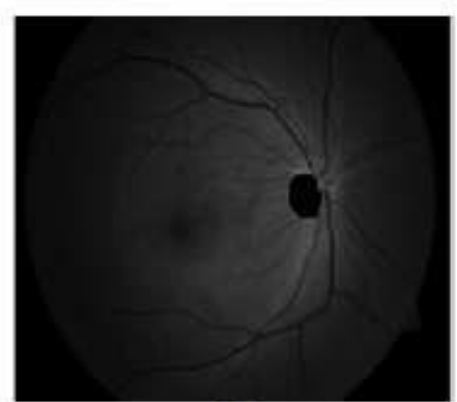

(b)

Figure 10. Optical Disc Detection (a) Original Images (b) Output Images 


\section{Conclusions and recommendations}

In this research, the performance of some methods of diagnosis of diabetic retinopathy was evaluated and a set of suggested steps for the detection of lesions of diabetic retinopathy and excretion through image processing techniques were applied. These procedures included FCM data collection algorithm, $\mathrm{H}$ and V channels of HSV format, standard deviation of the green channel of the retinal image, and the removal of hidden data from the image as a feature used in the FCM algorithm input. And removed from the picture Using its Alamamaat morphological Chapo chromatically with thousands of Arazat. The performance of the proposed steps was evaluated by some performance indicators such as positive predictive value (PPV), sensitivity, specificity and accuracy. The results showed that these steps depend on other functions such as optical disc detection. The set of steps will not work very efficiently and can use more than one optical disk to improve this function.

These steps performed well on retinal images that have bright and compact secretion areas and did not use vascular removal in this work, which can further improve system performance.

Combining FCM aggregation with another method or using supervised clustering method can lead to superior results

\section{References}

[1] N. J. Qasim and I. Barazanchi, "Unconstrained Joint Face Detection and Recognition in Video Surveillance System,” Jour Adv Res. Dyn. Control Syst., vol. 11, no. 1, pp. 1855-1862, 2019.

[2] C. SINTHANAYOTHIN, J.F. BOYCE, H.L. COOK and T.H. WILLIAMSON, "Automated location of the optic disk, fovea, \& retinal blood vessels from digital color fundus images," British Journal of Ophthalmology, Vol 83, pp. 902-910, 1999.

[3] A. SOPHARAK, K. THET NEW, Y. AYE MOE, M.N. DAILEY and B. UYYANONYARA. "Automatic exudate detection with a naive Bayes classifier," International Conference on Embedded Systems and Intelligent Technology, Bangkok, Thailand, 2008, pp.139-142

[4] M. NIEMEIJER, M.D. ABRAMOFF and B. VAN GINNEKEN, "Fast detection of the optic disc and fovea in color fundus photographs," Med. Image Anal, 2009, Vol 13, 859-870.

[5] S. Rashid, A. Ahmed, I. Al Barazanchi, A. Mhana, and H. Rasheed, "Lung cancer classification using data mining and supervised learning algorithms on multi-dimensional data set," Period. Eng. Nat. Sci., vol. 7, no. 2, pp. 438-447, 2019.

[6] "Automatic detection of diabetic retinopathy exudates from non-dilated retinal images using mathematical morphology methods," Computerized Medical Imaging and Graphics, 2008, Vol. 8, pp.720 727.

[7] A. SOPHARAK, B. UYYANONYARA and S. BARMAN, "Automatic Exudate Detection from Nondilated Diabetic Retinopathy Retinal Images Using Fuzzy C-means Clustering," 2009, Vol. 9, pp. 2148 2161.

[8] S. Rashid, A. Ahmed, I. Al Barazanchi, and Z. A. Jaaz, "Clustering algorithms subjected to K-mean and gaussian mixture model on multidimensional data set," Period. Eng. Nat. Sci., vol. 7, no. 2, pp. 448457, 2019.

[9] S. Q. Salih and A. Rahman A. Alsewari, "Solving large-scale problems using multi-swarm particle swarm approach,” Int. J. Eng. Technol., vol. 7, no. 3, pp. 1725-1729, 2018. 
[10] Z. Abdulelah Al-Sudani, S. Q. Salih, A. Sharafati, and Z. M. Yaseen, "Development of multivariate adaptive regression spline integrated with differential evolution model for streamflow simulation," J. Hydrol., vol. 573, no. 2, pp. 1-12, 2019.

[11] I. Al Barazanchi, S. A. Hamid, R. A. Abdulrahman, and H. Rasheed, "Automated telemedicine and diagnosis system ( ATDS ) in diagnosing ailments and prescribing drugs," Period. Eng. Nat. Sci., vol. 7, no. 2, pp. 888-894, 2019.

[12] J. M. Stewart, "New approaches in the diagnosis and management of diabetic retinopathy," Annals of Eye Science, vol. 4, pp. 17-17, Apr. 2019.

[13] Y. Sun, "The Neural Network of One-Dimensional Convolution-An Example of the Diagnosis of Diabetic Retinopathy,” IEEE Access, vol. 7, pp. 69657-69666, 2019.

[14] I. Qureshi, J. Ma, and Q. Abbas, "Recent Development on Detection Methods for the Diagnosis of Diabetic Retinopathy," Symmetry, vol. 11, no. 6, p. 749, Jun. 2019.

[15] L. Brazionis, A. Jenkins, A. Keech, C. Ryan, A. Brown, J. Boffa, and S. Bursell, "Diabetic retinopathy in a remote Indigenous primary healthcare population: a Central Australian diabetic retinopathy screening study in the Telehealth Eye and Associated Medical Services Network project," Diabetic Medicine, vol. 35, no. 5, pp. 630-639, Feb. 2018.

[16] D. H. Patel, "Correlation of diabetic retinopathy with duration of Diabetes mellitus," Journal of Medical Science And clinical Research, vol. 6, no. 12, Dec. 2018.

[17] K. D. Lestari, I. P. Budhiastra, and A. A. A. Sukartini Djelantik, "Non Prolipherative Diabetic Retinopathy Has Similar Platelet Morphology with Prolipherative Diabetic Retinopathy," Bali Journal of Ophthalmology, vol. 2, no. 1, Feb. 2018.

[18] Ç. İlhan, "The Optimal Cutoff Value of Neutrophil/Lymphocyte Ratio in Severe Grades of Diabetic Retinopathy Short Title: NLR in Diabetic Retinopathy,” Beyoglu Eye Journal, 2019.

[19] K. Kumar V, “Ayurvedic Treatment of Diabetic Retinopathy- A Review," International Journal of Ayurvedic and Herbal Medicine, Jun. 2018. 